\title{
Study on Noise Reduction Using a Wavelet Packet for Ultrasonic Flaw Detection Signal of a Small Diameter Steel Pipe with a Thick Wall
}

\author{
Yongqiao Wei \\ School of Manufacturing Science and Engineering, Sichuan University (610065), Chengdu, China \\ Rui Tang \\ School of Manufacturing Science and Engineering, Sichuan University (610065), Chengdu, China \\ School of Mechanical and Electrical Engineering, Panzhihua University (617000), Panzhihua,China \\ Li Hou, Fenglan Jia, Zhijun Sun and Bo Li \\ School of Manufacturing Science and Engineering, Sichuan University (610065), Chengdu, China
}

\begin{abstract}
(Received 3 May 2013; revised 7 October 2013; accepted 3 March 2014)
Ultrasonic detection technology is an important tool that is used to ensure the safe operation of equipment. However, when carrying out ultrasonic testing, especially in the ultrasonic detection of small-diameter steel pipes with thick walls, a mass of structural noise causes the useful information to get lost. This is problematic because the quality of ultrasonic echo signals is the foundation for the pipeline flaw-identification and feature extraction. At present, the wavelet packet thresholding method is the most widely used in signal de-noising processes. In this paper, a de-noising method of a wavelet packet based on the threshold is proposed for the limitation of both soft and hard thresholds. The ultrasonic wave pipeline detection signal that is often contaminated by noise is de-noised by this method. Moreover, the contrastive analysis is performed among the wavelet packet transform based on an adjusted threshold, the original ultrasonic echo signal, and the default threshold. Experiment results show that the noise reduction effect of the wavelet packet based on the adjusted threshold is the most reasonable one, and the signal-to-noise ratio (SNR) of the signals is also improved.
\end{abstract}

\section{INTRODUCTION}

With the wide application of small-diameter steel pipes with thick walls that transport high-temperature or high-pressure fluid, it is critical to ensure that small-diameter steel pipes with thick walls have high intensity and high quality. ${ }^{1}$ When detecting the small-diameter steel pipes with thick walls using the ultrasonic contact technique, their small diameter and high curvature leads to a small contact surface of the probe and tubing, large wear, poor coupling, serious proliferation of wave beams, and low examination-sensitivity. ${ }^{2}$ Seamless steel pipes, working under the situation of high temperature and high pressure, have the diameter of approximately $10 \mathrm{~mm}-3000 \mathrm{~mm}$ and a wall thickness of approximately $2 \mathrm{~mm}-60 \mathrm{~mm} .{ }^{3}$ Because of these rather harsh working conditions and high quality requirements, it is necessary to do non-destructive testing.

The detection of small-diameter steel pipes with a thick wall usually requires ultrasonic-testing technology, which is extensively applied in the field of non-destructive testing technology. ${ }^{2,4}$ Ultrasonic testing, a non-destructive type of testing for metals as well as non-metallic and composite materials, has strong penetration, high sensitivity, low cost, high efficiency, and non-contact detection. It is also an important direction for pipeline flaw detection. ${ }^{4,5}$

However, due to the multi-modal characteristics and dispersion phenomena of the ultrasonic wave, the received flaw-echo signal is relatively weak. When the ultrasonic wave is used for the actual flaw detection, the signal is inevitably disturbed by noise, and the difficulty of extraction and recognition for the flaw-echo wave signal is increased. Therefore, it is neces- sary for the ultrasonic echo signals that have been detected to receive de-noise pre-treatment. ${ }^{6}$

Enhancement of the signal-to-noise ratio (SNR) by using various signal processing techniques has become surprisingly well developed. ${ }^{5-8}$ Signal time averaging, sparse deconvolution method, matched band-pass filtering, frequency spectrum analysis, autocorrelation analysis, high-order spectrum analysis, split spectrum processing technique (SSP), and autoregressive analysis have all been used to de-noise ultrasonic signals. ${ }^{7}$

At present, wavelet analysis is extensively applied in the field of ultrasonic signal de-noising. Threshold de-noising is a wavelet de-noising method that is simple and more effective, but the traditional soft and hard threshold de-noising methods still have some limitations. ${ }^{6,8}$ The discontinuity of the hard threshold function can make the de-noised signal generate oscillatory phenomena. Although the soft threshold function can overcome the discontinuity, the method always has a constant deviation; the de-noised signal has obvious distortion when the wavelet coefficient with the larger absolute value is reduced. In the wavelet analysis method, the signal is decomposed into different levels and different location wavelet components, and the senior and most senior mean square wavelet amplitude values are made the necessary extracted eigenvalues. ${ }^{9}$ However, the wavelet decomposition only decomposes the lowfrequency signal and cannot decompose the high-frequency signal; so, the high-frequency signal cannot be used, and the extracted signal is not comprehensive enough. The wavelet packet that is equivalent to both a low-pass filter and a highpass filter can decompose the low-frequency signal and the 